\title{
Diffusion Weighted Magnetic Resonance Imaging for the Characterization of Solitary Pulmonary Lesions
}

\author{
Çağlayan Çakır ${ }^{1}$, Hakan Gençhellaç², Osman Temizöz ${ }^{3}$, Ahmet Polat ${ }^{4}$, Ersin Şengül², \\ Gökhan Duygulu ${ }^{5}$
}

\author{
'Department of Radiology, Kocaeli Derince Training and Research Hospital, Kocaeli, Turkey \\ ${ }^{2}$ Department of Radiology, Trakya University Hospital, Edirne, Turkey \\ ${ }^{3}$ Department of Radiology, Selçuk University Hospital, İzmir, Turkey \\ ${ }^{4}$ Department of Radiology, Edirne State Hospital, Edirne, Turkey \\ ${ }^{5}$ Department of Radiology, İzmir Katip Çelebi University Atatürk Training and Research Hospital, İzmir, Turkey
}

Background: We evaluated the differential diagnosis of solitary pulmonary lesions on magnetic resonance imaging. Aims: To investigate the value of diffusion weighted imaging on the differential diagnosis of solitary pulmonary lesions.

Study Design: Randomized prospective study.

Methods: This prospective study included 48 solitary pulmonary nodules and masses (18 benign, 30 malignant). Single shot echo planar spin echo diffusion weighted imaging (DWI) was performed with two $b$ factors $\left(0\right.$ and $\left.1000 \mathrm{~s} / \mathrm{mm}^{2}\right)$. Apparent diffusion coefficients (ADCs) were calculated. On diffusion weighted (DW) trace images, the signal intensities (SI) of the lesions were visually compared to the SI of the thoracic spinal cord using a 5-point scale: 1: hypointense, 2: moderately hypointense, 3: isointense, 4: moderately hyperintense, 5: significantly hyperintense. For the quantitative evaluation, the lesion to thoracic spinal signal intensity ratios and the ADCs of the lesions were compared between groups.
Results: On visual evaluation, taking the density of the spinal cord as a reference, most benign lesions were found to be hypointense, while most of the malignant lesions were evaluated as hyperintense on DWI with a $b$ factor of $1000 \mathrm{~s} / \mathrm{mm}^{2}$. In contrast, on T2 weighted images, it was seen that the distinction of malignant lesions from benign lesions was not statistically significant. The ADCs of the malignant lesions were significantly lower than those of benign lesions (mean ADC was $2.02 \times 10^{-3} \mathrm{~mm}^{2} / \mathrm{s}$ for malignant lesions, and $1.195 \times 10^{-3} \pm 0.3 \mathrm{~mm}^{2} / \mathrm{s}$ for benign lesions). Setting the cut-off value at $1.5 \times 10^{-3}$, ADC had a sensitivity of $86.7 \%$ and a specificity of $88.9 \%$ for the differentiation of benign lesions from malignant lesions. Conclusion: DWI may aid in the differential diagnosis of solitary pulmonary lesions. (ClinicalTrials.gov Identifier: NCT02482181)

Keywords: $b$ factor, diffusion weighted magnetic resonance imaging, lung cancer, solitary pulmonary nodule
When a patient is diagnosed with a lung lesion, the differential diagnosis is important, since the treatment is determined by the characteristics of the lesion. The goal in the evaluation of solitary pulmonary lesions is to distinguish malignant lesions from benign lesions in as non-invasive a manner as possible. As computed tomography (CT) has become widely used, the diagnosis of solitary pulmonary lesions has become easier. The size, features of the edges, shape, internal structure (calcification, fat content, cavitation, etc.), density, satellite nodules, growth rate and contrast involvement of the lesion 
are important properties which help to distinguish benign lesions from malignant ones. However, as the distinction is not absolute at all times, other imaging methods such as positron emission tomography and magnetic resonance imaging are preferred. The importance of this issue is the high five-year survival rate in early-diagnosed lung cancer cases (1).

Diffusion is the randomized microscopic motion of water molecules. It is known that diffusion is a sensitive parameter of tissue characterization at a microscopic level. Nowadays, diffusion is measured in vivo with diffusion weighted imaging (DWI) and ADC measurements (2).

Diffusion weighted imaging has a wide use on oncologic patients for the purpose of diagnosis. In addition, it is used in the distinction of acute cerebral infarction (3) and epidermoid or arachnoid cysts (4). Recently, it has also been used in the characterization of cystic or solid lesions in the thoracic cavity. In this study, we aimed to evaluate the accuracy of differentiation of solitary pulmonary lesions.

\section{MATERIALS AND METHODS}

\section{Patients}

Approval for the study was granted by the Institutional Review Board and the Protocol Examination Committee. Written informed consent was obtained from all patients. Magnetic resonance imaging (MRI) was applied to a total of 46 patients between January 2011 and July 2011, who presented at our department for thoracic CT for various reasons. The patients included 33 males and 13 females aged 20-78 years. All patients had a solitary pulmonary nodule or mass. The study comprised 48 lesions of 46 patients, who could be contacted with pathology results and could be diagnosed with follow-up results.

Of the studied lesions, 30 were malignant masses and 18 were benign pathologies. Of these malignant masses, 21 $(43.75 \%)$ were primary lung tumors and nine $(18.75 \%)$ were metastatic lung nodules confirmed by biopsy or pathology results following surgery. One of the lesions disappeared as a result of antibiotic therapy, so was classified as pneumonia. In addition, the diagnoses of five hydatid cyst cases were confirmed by clinical and laboratory results.

Two cases of tuberculoma were confirmed by final pathological assessment after surgical treatment. The other 10 benign nodules or masses which did not grow during the follow-up period were classified as granulomas, fibrous nodules, bronchogenic cysts and atelectasis.

In the T1-weighted axial images, the diameter was calculated using the average of the long and short axis diameters of the nodule/mass. The average diameter of all lesions was found to be $27.4 \mathrm{~mm}$. Based on lesions which were examined in the study, it was found that the average diameter of malignant lesions was greater than that of benign lesions.

\section{MR imaging}

All patients were examined with a 1.5 Tesla MR scanner (General Electric Medical Systems, Signa HDxt GE GEHC Excite II, Milwaukee USA) using an eight-element phasedarray body coil. This system has a maximal gradient strength of $32 \mathrm{mT} / \mathrm{m}$ and a slew rate of $120 \mathrm{mT} / \mathrm{m} / \mathrm{ms}$. All patients were examined initially with non-contrast axial $\mathrm{T} 1$ weighted (W) breath hold spoiled (Time to repeat (TR)/Time to echo (TE)/ Spin echo/Number of excitations (NEX), 169/5/80/4), coronal and axial T2W single shot fast spin echo (SSFSE) (TR/TE/ NEX/Fast Spin Echo factor, 700/80/4/72). Subsequently, three series of axial single shot spin echo-echo planar (SS-SE-EP) images were examined.

Diffusion weighted images (TR/TE/NEX/echo-planar imaging factor, 4800/68/4/77; sensitizing gradients in $\mathrm{x}, \mathrm{y}, \mathrm{z}$ directions) were acquired using the following $b$ values: 0.50 , 500 , and $1000 \mathrm{~s} / \mathrm{mm}^{2}$. MRI, including DWI, consisted of a multisection acquisition with a slice thickness of $5.5 \mathrm{~mm}$ and an acquisition matrix of $128 \times 128$. The field of view varied between 370 and $400 \mathrm{~mm}$.

The images obtained from patients were transferred to a separate work station, the Advantage Workstation 4.1 (General Electric Medical Systems, Milwaukee, USA). ADC maps were created with image processing software (Functool). An ADC map of each slice was created.

\section{MR imaging analysis}

\section{Qualitative analysis}

In the MR unit, two radiologists with 14 and 4 years of experience evaluated solitary lesion signal intensity on a scale of 1 to 5 by diffusion and T2-weighted images in the transverse plane. The radiologists were blind to the CT findings and the clinical information of the patients. Comments were anonymous, but in three cases, the final judgment was reached by discussion.

As the DWI signals were affected by both $\mathrm{T} 1$ and $\mathrm{T} 2$ relaxation times and spin density, the signal intensities (SI) in DWI which were obtained only with a $b$ factor of $1000 \mathrm{~s} / \mathrm{mm}^{2}$ as it was found to be suitable for evaluation. Therefore, especially with low $\mathrm{b}$ factors, the lesions showed a T2 smear effect rather than a lack of diffusion.

Two radiologists independently reviewed the signal intensity of the nodules on DWI with a $b$ factor of $1000 \mathrm{~s} / \mathrm{mm}^{2}$ using a 5 -point rank scale. Intensities were rated on a scale from 1 to 5: 1 , no signal intensity; 2 , signal intensity between 1 and $3 ; 3$, signal intensity similar to thoracic spinal cord intensity; 4 , high- 
er signal intensity than the spinal cord intensity; and 5, much higher signal intensity than the thoracic spinal cord intensity. Wang et al. (5) suggested using the spinal cord instead of physiological saline as a reference in diffusion weighted imaging. In this study, the spinal cord was taken as the reference $(5,6)$.

The scale for T2-weighted images was based on the following scores: 1 , nearly no signal intensity, hypointense; 2 , signal intensity almost equal to that of the dorsal muscles; 3 , lower signal intensity between 2 and 5; 4, higher signal intensity between 2 and 5 ; and 5, signal intensity almost equal to that of cerebral spinal fluid (CSF) in the thoracic spine.

\section{Quantitative analyses}

Quantitative analyses were performed using a workstation (Advantage Workstation system 4.1 inches). ADC maps were created with image processing software (Functool). For digital evaluation, ADC was measured by the $b$ factor $\left(1000 \mathrm{~s} / \mathrm{mm}^{2}\right)$ obtained by DWI from the ADC, using a region of interest (ROI) and map images of the lesion (at least two thirds of the tray). To prevent possible artifacts, signal intensity (SI) measurements and the determination of ADC values were performed as far away as possible from the diaphragm. Due to the limited resolution of DWI, lesions smaller than $5 \mathrm{~mm}$ were not taken into consideration. The final diagnosis in most of the lesions was confirmed by pathological evaluation.

\section{Statistical analysis}

Statistical analysis of the data was analyzed by the one-sample Kolmogorov Smirnov test for a normal distribution. To verify the normal distribution, the Mann Whitney U test was used. The Yates-corrected $\chi 2$ test was used for qualitative data. The mean \pm standard deviation and median (min-max) values were given as descriptive statistics. ROC analysis was performed for the specificity and sensitivity of the ADC value. A value of $p<0.05$ was accepted as statistically significant. The statistical analyses were performed with SPSS (SPSS Inc., Chicago, USA).

\section{RESULTS}

\section{Qualitative analysis}

The diagnoses of the 48 lesions with diffusion-weighted imaging scores are listed in Table 1 . Thirty nodules in 28 patients were malignant in nature and 18 lesions in 18 patients were benign lesions.

On DWI, $16 / 18$ benign lesions were scored as $\leq 2$, and two tuberculoma cases were scored as 5 by the radiologists. Of the malignant lesions, 28/30 were scored as $\geq 3$ and two cases of metastatic lung nodules were scored as 2 by the radiologists.
In the benign group, $10 \%(2 / 18)$ had overlapping scores with the malignant group and $6.6 \%(2 / 30)$ of the malignant group overlapped with the benign group.

The scores on the 5-point rank scale used to evaluate malignant and benign pulmonary lesions on DW images were $4.03 \pm 0.999$ and $2.33 \pm 0.970$, respectively. On DW images, the scores on the 5-point rank scale of malignant pulmonary lesions were significantly higher than those of benign lesions $(p<0.05)$ and the area under the receiver operating characteristic (ROC) curve was 0.921 .

When scores of $\geq 3$ were accepted as malignant and scores of 1 and 2 as benign, the sensitivity and specificity were $93.3 \%$ and $88.9 \%$, respectively.

On T2-weighted images, the scores on the 5-point rank scale of malignant pulmonary nodules $(3.12 \pm 0.539)$ were not significantly higher $(p>0.05)$ than those of benign nodules (2.91 \pm 0.818$)$. Samples from both groups of images of the lesions are shown in Figure 1 and 2.

\section{Quantitative analysis}

The results of quantitative analysis of the data obtained from the two disease groups are shown in Table 2. When the cut-off value was $1.5 \times 10^{-3}$, based on the results of the evaluation of ADC values obtained from DW images, the sensitivity was $86.67 \%$ and specificity was $88.89 \%$. The positive predictive value was $92.9 \%$ and the negative predictive value was $80.0 \%$ (Figure 3). There was no significant difference between signal intensity rates and $\mathrm{ADC}$ values in the subgroup analyses.

TABLE 1. Diagnoses and scores of pulmonary lesions (nodules and masses) evaluated with diffusion-weighted imaging

\begin{tabular}{|c|c|c|c|c|c|c|}
\hline \multirow{2}{*}{$\begin{array}{l}\text { Diagnosis of pulmonary } \\
\text { nodule/mass }\end{array}$} & \multicolumn{5}{|c|}{ 5-Point Rank Scale } & \multirow[b]{2}{*}{ TOTAL } \\
\hline & 1 & 2 & 3 & 4 & 5 & \\
\hline Primary lung cancer & & & 6 & 6 & 9 & 21 \\
\hline Metastatic lung cancer & & 2 & 2 & 5 & & 9 \\
\hline Fibrous nodule & & 4 & & & & 4 \\
\hline Round atelectasis & & 2 & & & & 2 \\
\hline Tuberculoma & & & & & 2 & 2 \\
\hline Pneumonia & & 1 & & & & 1 \\
\hline Abscess & & 1 & & & & 1 \\
\hline Granuloma & & 1 & & & & 1 \\
\hline Hydatid cyst & & 5 & & & & 5 \\
\hline Bronchogenic cyst & & 1 & & & & 1 \\
\hline Phantom tm & & 1 & & & & 1 \\
\hline TOTAL & 0 & 18 & 8 & 11 & 11 & 48 \\
\hline
\end{tabular}

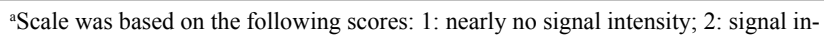
tensity between 1 and $3 ; 3$ : signal intensity almost equal to that of the thoracic spinal cord; 4: higher signal intensity than that of the spinal cord; and 5: much higher signal intensity than that of the spinal cord. 

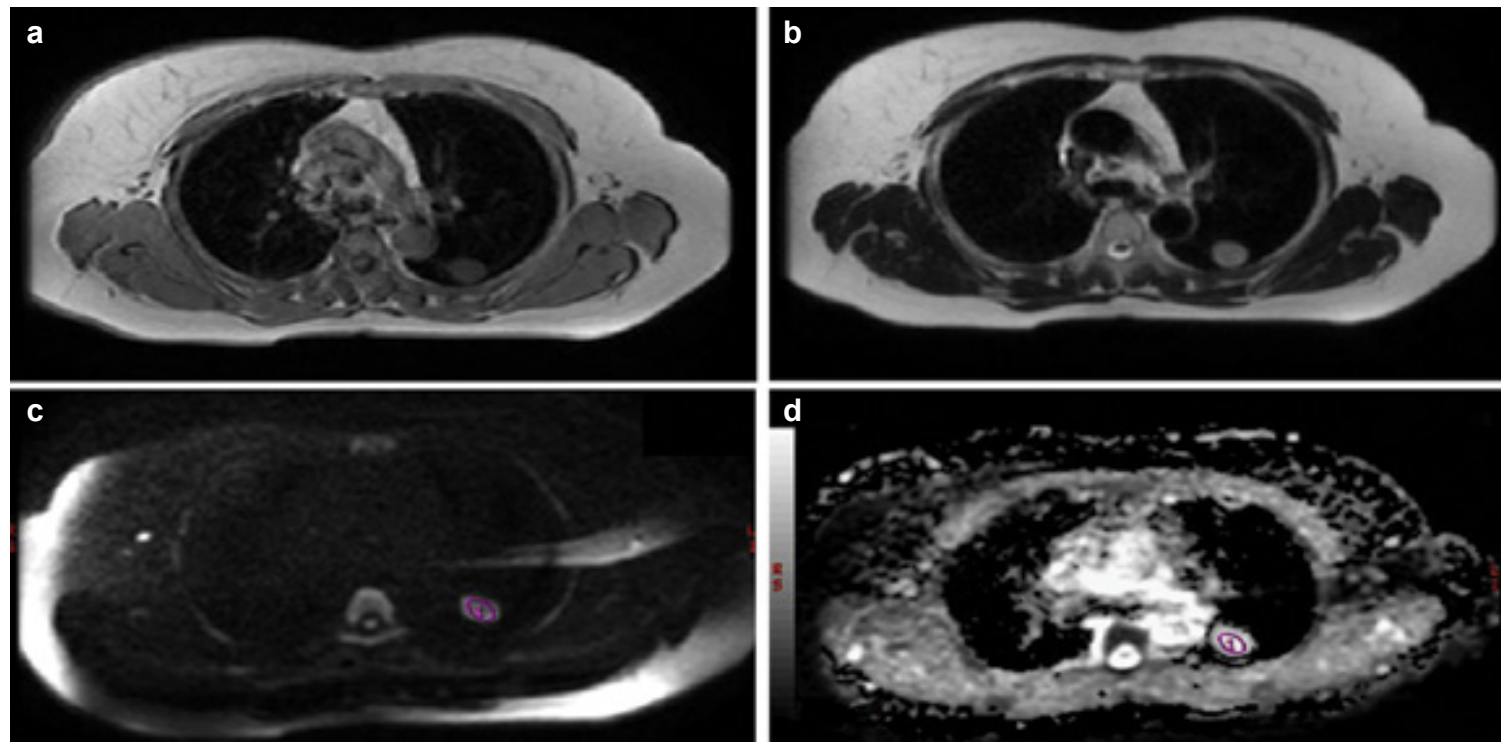

FIG. 1. a-d. Axial T1 weighted image shows a nodule in the posterior left upper lobe (a), axial T2 weighted image shows a hypointense nodule in the posterior left upper lobe (b), axial diffusion weighted image obtained with $a b$ factor of $1000 \mathrm{~s} / \mathrm{mm}^{2}$ shows a nodule with low signal intensity compared with the spinal cord (c); it scored 2 on the 5-point rank scale. The spinal cord scored 3 on the 5-point rank scale on DW images obtained with $a b$ factor of $1000 \mathrm{~s} / \mathrm{mm}^{2}$. Axial apparent diffusion coefficient value of the lesion $\left(2.190 \times 10^{-3}\right)(d)$, when the $b$ factor was taken as $b=1000$ $\mathrm{mm}^{2} / \mathrm{s}$; this was higher than our cut-off value $\left(1.5 \times 10^{-3}\right)$.

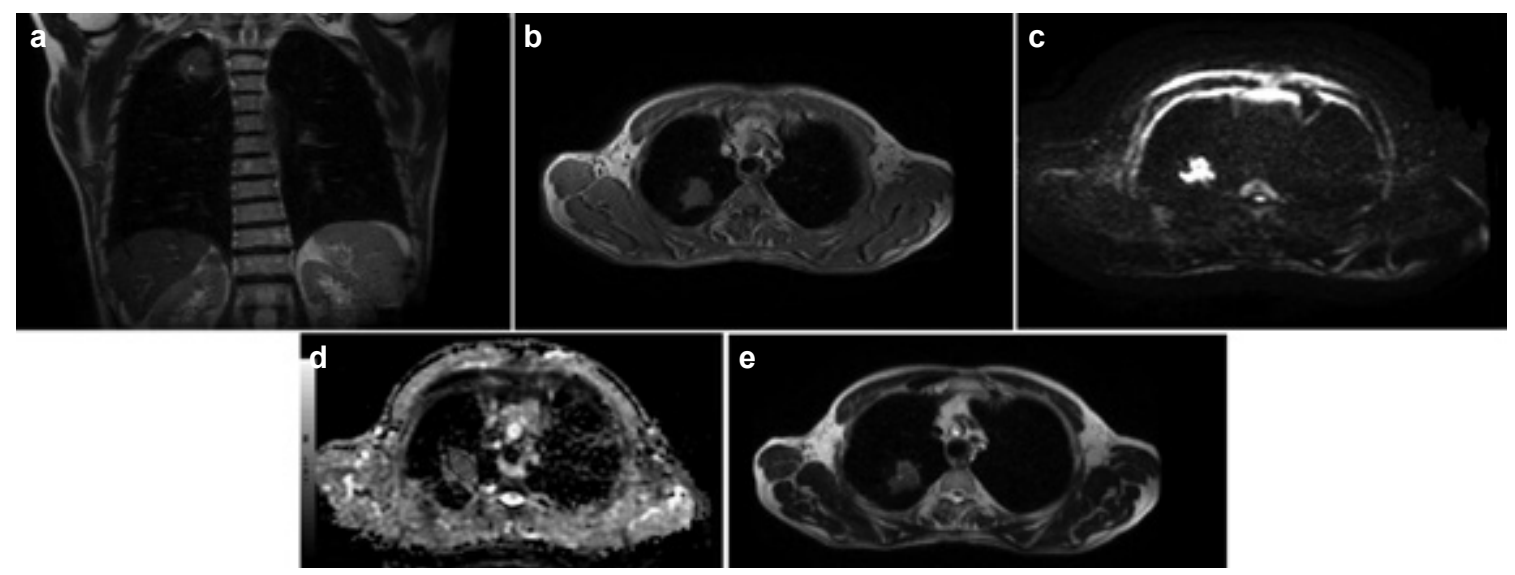

FIG. 2. a-e. Axial (a) and coronal (b) T2-weighted echo-planar image obtained with a b factor of $0 \mathrm{~s} / \mathrm{mm}^{2}$ shows a mass with a slightly lower signal intensity compared with the CSF and a high signal intensity compared with the dorsal muscle; it scored 4 on the 5-point rank scale. CSF scored 5 on the 5-point rank scale on the T2-weighted image obtained with a b factor of $0 \mathrm{~s} / \mathrm{mm}^{2}$. The dorsal muscle scored 2 on the 5 -point rank scale on T2-weighted images obtained with a $b$ factor of $0 \mathrm{~s} / \mathrm{mm}^{2}$.

Axial T1-weighted image shows a mass in the right upper lobe (c), axial diffusion weighted echo-planar image obtained with a b factor of $1000 \mathrm{~s} /$ $\mathrm{mm}^{2}$ shows a mass with very high signal intensity compared with the spinal cord (d); it scored 5 on the 5-point rank scale. The spinal cord scored 3 on the 5-point rank scale on DW images obtained with a b factor of $1000 \mathrm{~s} / \mathrm{mm}^{2}$, axial apparent diffusion coefficient value of the lesion (1.05x10$\left.{ }^{3}\right)(e)$, when the $b$ factor was taken as $b=1000 \mathrm{~mm}^{2} / \mathrm{s}$; this was lower than our cut-off value $\left(1.5 \times 10^{-3}\right)$.

\section{DISCUSSION}

The use of DWI in the chest cavity is limited because of the artifacts caused by respiratory or cardiac motion. The effects of respiratory and cardiac motion can be removed by holding the breath and/or using a single-shot sequence. The best image quality is obtained by taking SS-SE-EPI DWI long with holding the breath because of high recovery capacity and high signal/noise ratio (7). In the current study, DWI were obtained by EPI, which provided a short echo time of sequence and STIR and SENSE. DWI signals are affected by both T1 and $\mathrm{T} 2$ relaxation times and the spin density. Therefore, lesions may show a strong T2 effect instead of lack of diffusion, especially with low $b$ factors (shine effect of T2). Diffusion can 


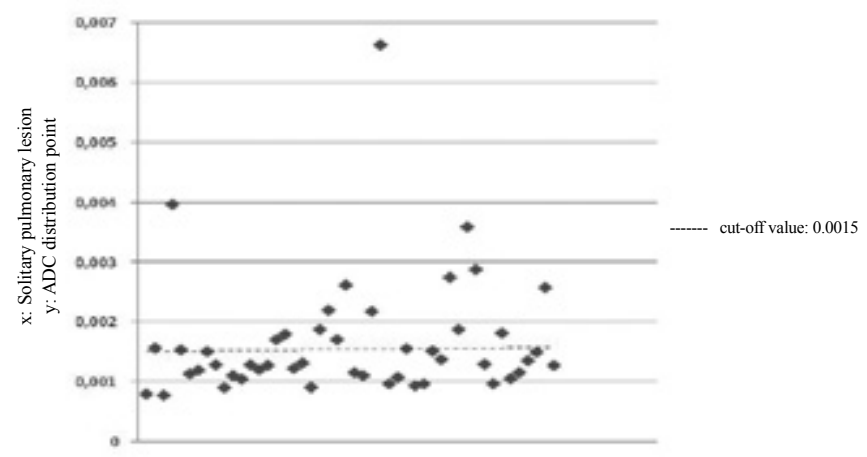

FIG. 3. The apparent diffusion coefficient distribution point in the graph

TABLE 2. Groups of patients according to disease apparent diffusion coefficient value scores (b 1000)

\begin{tabular}{lccc}
\hline & Malign $(\mathrm{n}=30)$ & Benign $(\mathrm{n}=18)$ & \\
\hline b 1000 mean ADC & $1.185 \pm 0.236$ & $2.423 \pm 1.299$ & \\
$\left(\mathrm{~mm}^{2} / \mathrm{s}\right) 10^{-3}$ & $1.195(0.771-1.700)$ & $2.02(1.10-6.62)$ & $\mathrm{p}<0.05$ \\
\hline
\end{tabular}

$\mathrm{x} \pm \mathrm{ss}$ : average \pm standard deviation; ADC: apparent diffusion coefficients

Mann-Whitney U test

be evaluated quantitatively with ADC independent of the T2 shine effect, as was the case in the current study $(8,9)$.

In a study by Mayo et al. (10), a comparison was made between normal and abnormal lung parenchyma with high-resolution CT using the short echo delayed ( $\mathrm{TE}=7 \mathrm{~ms}) \mathrm{T} 1$-weighted sequence and the conventional $(\mathrm{TE}=20 \mathrm{~ms}) \mathrm{T} 1$-weighted sequence. It was determined that, when split into short sequences, the SNR increased significantly compared to conventional sequences and better images were obtained of normal and abnormal lung parenchyma. Despite less anatomical detail than CT, it has been proposed that MRI could be used for the follow-up of parenchymal lung disease. In the current series, the characterization of solitary pulmonary lesions, benign and malignant differentiation, by the visual assessment of SI was not found to be statistically significant.

In a meta-analysis, Schaefer et al. (11) proposed a sensitivity of $93.9 \%$ and a specificity of $93.9 \%$ in an evaluation obtained from dynamic MR images, in solitary pulmonary nodules detected by dynamic contrast $\mathrm{CT}$ in benign and malignant differentiation. Hittmair et al. (12), Guckel et al. (13) and Ohno et al. (14) reached higher specificity in similar studies on the differentiation of benign and malignant solitary pulmonary lesions with dynamic MRI. In the current study, similar results were also obtained in the evaluation according to SI in DWI, which were intended for solitary pulmonary lesions. Therefore, it can be considered that where the use of contrast agents is contra-indicated (for example, in cases with chronic renal failure), adding diffusion weighted images to the routine thoracic MRI examination of the patient may be beneficial to the radiologist in identifying the characteristics of the lesion.
Hatabu et al. (15) used single-shot turbo spin-echo (HASTE) sequences for the evaluation of lung parenchyma and correlated pathologies with MRI in 20 patients. The images were obtained with ECG-triggered and with breath-holding, and they observed that cardiac and respiratory artifacts were minimal. HASTE sequences were reported to successfully diagnose lung cancer, hilar lymphadenopathy, metastatic lesions up to 3 $\mathrm{mm}$, pulmonary hemorrhage, edema and bronchial wall thickening in bronchiectasis. Similar findings have been observed in several studies with other imaging techniques $(16,17)$.

İnan et al. (18) showed the results of the visual evaluation of the SI of pleural effusions in diffusion trace images with $b$ factors of 500 and $1000 \mathrm{~s} / \mathrm{mm}^{2}$. In these images, the SI of exudative pleural effusions were clearly higher than those of transudative pleural effusions. Most of the transudative pleural effusions were nearly isointense, while most of the exudative pleural effusions were hyperintense. In our study, we independently reviewed the signal intensity of the lesions on DWI with $\mathrm{b}$ factor of $1000 \mathrm{~s} / \mathrm{mm}^{2}$ using a 5-point rank scale. When scores of $\geq 3$ were accepted as malignant and scores of $\geq 2$ as benign, the sensitivity was $93.3 \%$ and specificity was $88.9 \%$.

Satoh et al. (19) designed a study similar to the current one to diagnose pulmonary lesions $<5 \mathrm{~mm}$, and compared the pathological results of the lesions. In DWI obtained with $a b$ factor of $1000 \mathrm{~s} / \mathrm{mm}^{2}$, as in the current study, scoring was applied according to the signal intensity of the lesion by taking the spinal cord as a reference; the SI of malignant lesions were reported to be higher than those of benign lesions. The area under the ROC curve was found to be 0.87 , similar to that of the current study (ROC value: 0.921 ).

Satoh et al. (19) applied visual scoring to T2-weighted images in a manner similar to the current study, but no difference was determined between the SI of malignant and benign lesions. In the current study, similar results were obtained in the visual distinction of lesions on T2-weighted images.

Satoh et al. (19) evaluated qualitative analyses of diffusionweighted and T2-weighted images of the lesions, not taking ADC values into account due to the extensive movement of artifacts (heart rate, breathing). In a study by Matoba et al. (20), the ADCs of lung carcinomas correlated with tumor cellularities. The ADCs of well-differentiated adenocarcinoma appear to be higher than those of other lung carcinoma types (squamous cell carcinoma and large-cell carcinoma). In the current study, ADC mapping (Functool) was obtained with breath-holding DWI, and after the evaluation of the data, it was seen to be useful in diagnosing solitary pulmonary nodules and masses. When the cut-off value was $1.5 \times 10^{-3}$, based on the results of the evaluation of ADC values obtained from DW images, the sensitivity was $86.67 \%$ and the specificity was $88.89 \%$. 
There are some limitations to our study. First of all, a high $b$ value ( $b=1000)$ MR diffusion sequence was used, but because of the wide range of lesions to be identified and excessive brightness, scoring with DWI obtained with lower $b$ factors $\left(50\right.$ and $500 \mathrm{~s} / \mathrm{mm}^{2}$ ) could not be performed. Images obtained with $\mathrm{a} b$ factor of $1000 \mathrm{~s} / \mathrm{mm}^{2}$ were found to be optimal for evaluation, and the lesions were found to be suitable since they indicated significantly different SI. Another limitation, cardiac artifacts, could not be blocked. Therefore, pulmonary lesions smaller than $5 \mathrm{~mm}$ were not included in our study. Despite everything, while metastatic lesions and malignant lesions such as adenocarcinoma were isointense or hyperintense compared to the spinal cord, benign lesions seemed to be isointense. Further research on this subject is required.

Pulmonary lesions are currently diagnosed using radiological findings (size, edge features, shape, internal structure features, contrast enhancement, growth rate, satellite nodules), laboratory results and clinical assessments. However, it can sometimes be difficult to distinguish benign from malignant lesions, especially on CT, requires the use of non-ionizing radiation.

Magnetic resonance imaging, which does not involve nonionizing radiation, is a very safe imaging method. Suitable protocols should be prepared for suspected patients. Lately, MRI has become an important radiological imaging technique used in the diagnosis of lung pathologies with breath holding rapid sequences which reduce cardiac motions and respiratory artifacts. Diffusion MRI with ADC value measurement as a functional imaging method may be a tool for diagnosis or differential diagnosis, but it is far from being the gold standard (21). With reasonable sensitivity and specificity, no requirement for contrast material and easy application, the procedure may be added to the routine protocol. To confirm these findings, prospective randomized trials should be performed on larger populations.

Ethics Committee Approval: Ethics committee approval was received for this study from the ethics committee of Trakya University Ethics Committee.

Informed Consent: Written informed consent was obtained from patients who participated in this study.

Peer-review: Externally peer-reviewed.

Author contributions: Concept - Ç.Ç., H.G., O.T.; Design - Ç.Ç., H.G., O.T.; Supervision - Ç.Ç., H.G., O.T., A.P., E.Ş., G.D.; Resource - Ç.Ç., H.G., O.T.; Materials - Ç.Ç., A.P., E.Ş.; Data Collection \&/ or Processing - Ç.Ç., H.G., O.T., A.P., E.Ş., G.D.; Analysis \&/or Interpretation - Ç.Ç., H.G., O.T., A.P., E.Ş., G.D.; Literature Search - Ç.Ç., H.G., O.T., A.P., E.Ş., G.D.; Writing - Ç.Ç., H.G., O.T., A.P., E.Ş., G.D.; Critical Reviews - Ç.Ç., H.G., O.T., A.P., E.Ş., G.D.
Conflict of Interest: No conflict of interest was declared by the authors.

Financial Disclosure: Our study supported financially by Trakya University Hospital.

\section{REFERENCES}

1. Swensen SJ, Brown LR, Colby TV, Weaver AL. Pulmonary nodules: CT evaluation of enhancement with iodinated contrast material. Radiology 1995;194:393-8. [CrossRef]

2. Le Bihan D, Turner R, Douek P, Patronas N. Diffusion MR imaging: clinical applications. AJR Am J Roentgenol 1992;159:591-9. [CrossRef]

3. Ozsunar Y, Sorensen AG. Diffusion- and perfusion-weighted magnetic resonance imaging in human acute ischemic stroke: technica considerations. Top Magn Reson Imaging 2000;11: 259-72. [CrossRef]

4. Hakyemez B, Yildiz H, Ergin N, Uysal S, Parlak M. FLAIR and diffusion weighted MR imaging in differentiating epidermoid cysts from arachnoid cysts. Tani Girisim Radyol 2003;9:418-26.

5. Wang J, Takashima S, Takayama F, Kawakami S, Saito A, Matsushita T, et al. Head and neck lesions: characterization with diffusion-weighted echo-planar MR imaging. Radiology 2001;220:621-30. [CrossRef]

6. Tanner SF, Ramenghi LA, Ridgway JP, Berry E, Saysell MA, Martinez D, et al. Quantitative comparison of intra brain diffusion in adults and preterm and term neonate and infants. AJR Am J Roentgenol 2000;174:1643-9. [CrossRef]

7. Naganawa S, Kawai H, Fukatsu H, Sakurai Y, Aoki I, Miura S, et al. Diffusion-weighted imaging of the liver: technical challenges and prospects for the future. Magn Reson Med Sci 2005;4: 175-86. [CrossRef]

8. Purroy F, Begue R, Gil MI, Quílez A, Sanahuja J, Brieva L, et al. Patterns of diffusion-weighted magnetic resonance imaging associated with etiology improve the accuracy of prognosis after transient ischaemic attack. Eur J Neurol 2011;18:121-8. [CrossRef]

9. Hakyemez B, Aksoy U, Yildiz H, Ergin N. Intracranial epidermoid cysts: diffusion weighted, FLAIR and conventional MR findings. Eur J Radiol 2005;54:214-20. [CrossRef]

10. Mayo JR, MacKay A, Muller NL. MR imaging of thelungs: value of short TE spin-echopulse sequences. AJR Am J Roentgenol 1992;159:951-6. [CrossRef]

11. Schaefer JF, Vollmar J, Schick F, Vonthein R, Seemann MD, Aebert H, et al. Solitary pulmonary nodules: dynamic contrast enhanced MR imaging perfusion differences in malignant and benign lesions. Radiology 2004;232:544-53. [CrossRef]

12. Hittmair K, Eckersberger F, Klepetko W, Helbich T, Herold CJ. Evaluation of solitary pulmonary nodules with dynamic contrast enhanced MR imaging: a promising technique. Magn Reson Imaging 1995; 13:923-33. [CrossRef]

13. Guckel C, Schnabel K, Deimling M, Steinbrich W. Solitary pulmonary nodules: MR evaluation of enhancement patterns with 
contrast-enhanced dynamic snap shot gradient-echo imaging. Radiology 1996;200:681-6. [CrossRef]

14. Ohno Y, Hatabu H, Takenaka D, Adachi S, Kono M, Sugimura K. Solitary pulmonary nodules: potential role of dynamic MR imaging in management initial experience. Radiology 2002;224:503-11. [CrossRef]

15. Hatabu H, Stock KW, Chen Q, Edelman RR. Magnetic resonance T2 measurements of the normal human lung in vivowith ultra-short echotimes. Magn Reson Imaging 1999;17: 997-1000. [CrossRef]

16. Yi CA, Lee KS, Kim B, Choi JY, Kwon OJ, Kim H, et al. Tissue characterization of solitary pulmonary nodule: comparative study between helical dynamic CT and integrated PET/CT. J Nucl Med 2006;47:443-50.

17. Shim SS, Lee KS, Kim B, Choi JY, Chung MJ, Lee EJ. Focal parenchymal lung lesions showing a potential of false-positive and false-negative interpretations on integrated PET/CT. AJR Am J Roentgenol 2006;186:639-48. [CrossRef]

18. İnan N, Arslan A, Akansel G, Arslan Z, Eleman L, Demirci A. Diffusion-weighted MRI in the characterization of pleural effusions. Diagn Interv Radiol 2009;15:13-8.

19. Satoh S, Kitazume Y, Ohdama S, Kimula Y, Taura S, Endo Y. Can Malignant and Benign Pulmonary Nodules Be Differentiated with Diffusion-Weighted MRI? AJR Am J Roentgenol 2008;191:464-70. [CrossRef]

20. Munetaka M, Hisao T, Tamaki K, Hajime Y, Kotaro H, Hirohisa $\mathrm{T}$, et al. Lung Carcinoma: Diffusion weighted MR Imaging Preliminary Evaluation with Apparent Diffusion Coefficient. Radiology 2007;243:570-7. [CrossRef]

21. Cova M, Squillaci E, Stacul F, Manenti G, Gava S, Simonetti G, et al. Diffusion-weighted MRI in the evaluation of renallesions: preliminary results. Br J Radiol 2004;77:851-7. [CrossRef] 